\title{
Variación del perfil de consumo de sustancias en la unidad de desintoxicación del Hospital Psiquiátrico de Madrid: 1994-1999
}

\author{
RESUMEN: El estudio compara los perfi- \\ les y patrones de consumo de los pacientes \\ ingresados en la Unidad de Desintoxica- \\ ción del Hospital Psiquiátrico de Madrid \\ durante los años 1.994 y el 1.999 . \\ PALABRAS CLAVE: patrones en el uso \\ de sustancias, programa de mantenimiento \\ con metadona, programas de reducción \\ de daño.
}

\begin{abstract}
The study compares the profile and drug use patterns among patients admitted to the Unit of Detoxification of the Psychiatric Hospital of Madrid during the years 1.994 and 1.999. KEY WORDS: drug-use trends, methadone maintenance treatment (MMT), harm reduction treatment.
\end{abstract}

\section{Introducción.-}

Los dispositivos de atención a drogodependientes del Estado español, como en otros lugares del mundo, se desarrollaron inicial y preferentemente para tratar a heroinómanos. Las unidades de desintoxicación hospitalaria fueron concebidas como un primer escalón para el paso a una vida libre de drogas, cuando la desintoxicación no era posible en un medio más normalizado. La observación de los resultados de aplicar los programas libres de drogas como única respuesta a las drogodependencias puso en evidencia la insuficiencia de tales abordajes para reducir el consumo y para acercar los usuarios a la red socio sanitaria. Además, a partir de los años 80, la extensión del SIDA y de otras enfermedades infecciosas entre los usuarios de drogas por vía parenteral obligó a pensar en formas de intervención que, además de abordar el problema de la dependencia, ayudaran a disminuir la morbilidad y mortalidad de esta población, hicieran más accesible la red asistencial y, en definitiva, mejoraran la calidad de vida de los usuarios y la salud pública. Estos programas, denominados de "reducción del daño" (1),(2), han modificado los patrones de consumo, han revolucionado el trabajo de los profesionales y la demanda de los consumidores.

Las unidades de desintoxicación hospitalaria no son, o no deberían ser, ajenas a esta evolución y cabría preguntarse por su futuro ante las nuevas demandas y a la vista de los más recientes datos epidemiológicos. Mientras que la demanda de tratamiento por drogodependientes se ha disparado en la Comunidad de Madrid y ha pasado de 4.327 
usuarios atendidos en 1994 a 16.393 en 1999, más de 7000 en PMM, el número de los que pasaron en ese mismo periodo por una Unidad de Desintoxicación Hospitalaria se ha reducido de 428 a 373 (3),(4). Por otra parte, también se observa un aumento significativo de la dependencia de cocaína y del abuso de alcohol, benzodiacepinas y cocaína (el registro de casos de la Agencia Antidroga sólo permite reseñar una droga principal de consumo y dos secundarias. Esto supone que muchos de los casos de uso de cocaína, alcohol y benzodiacepinas puedan tratarse de dependencia pero el registro no los cataloga como tal, porque habitualmente se confiere al consumo de opiáceo, cuando existe, el código de droga principal). En el año 1994 el 83\% de los usuarios del Plan Regional de Drogas consumían heroína como droga principal o secundaria, 83.7\% cocaína, $52.8 \%$ alcohol, 38.6\% benzodiacepinas y sólo existían 232 plazas de dispensación de metadona. En 1999, entre los usuarios de la Agencia Antidroga (organismo que sustituyó al Plan Regional), 85\% consumían heroína como droga principal o secundaria, $25 \%$ cocaína como droga principal y $90 \%$ como droga secundaria, 85\% alcohol, $57 \%$ benzodiacepinas y el número de plazas de metadona, más de 7000, se había multiplicado por 30. Es conocido y puesto de relieve en la literatura que los programas de mantenimiento con agonistas opiáceos permiten abandonar el consumo de heroína y mejorar la adaptación familiar y social de gran número de drogodependientes, pero se asocian con frecuencia al abuso de drogas no opiáceos, generalmente alcohol, cannabis, cocaína y benzodiacepinas (5-8).

Esta evolución de los patrones de consumo de drogas de abuso en la calle se ha visto reflejada en el perfil de pacientes atendidos en la Unidad de Desintoxicación del Hospital Psiquiátrico de Madrid. Sopelana et al (9) ya señalaban, en un estudio realizado en esta misma unidad entre 1985 y 1990, una progresiva diversificación y aumento del número y cantidad de sustancias consumidas asociadas a la heroína. A partir de 1998 en respuesta a la creciente demanda de desintoxicación de otras sustancias se ampliaron los perfiles de desintoxicación ya existentes añadiendo la desintoxicación de benzodiacepinas, cocaína, alcohol y metadona (teóricamente hasta 70 gr/día) tanto en usuarios orientados a programa libre de drogas como a programa de mantenimiento con metadona. Esta modificación de los criterios de ingreso creemos ha supuesto un cambio en las características sociodemográficas y sanitarias y del tratamiento de desintoxicación que reciben los pacientes que pasan por la unidad. Para comprobar esta hipótesis pusimos en marcha un estudio comparando todos los pacientes que pasaron por la unidad entre el 1 de marzo y el 30 de septiembre de 1994 y el mismo periodo de 1999. En ambos casos se recogieron datos sociodemográficos, sanitarios, historia toxicológica, sustancias consumidas en el mes previo, tratamiento farmacológico durante el ingreso, tiempo de desintoxicación y motivo del alta. Otros posibles cambios relacionados con la entrada de nuevos perfiles, como pueden ser el ambiente de la unidad y la conflictividad de los usuarios durante su estancia, resultan difíciles de medir, por lo que no se tuvieron en cuenta aunque también pensamos se han modificado y afectan a la evolución del tratamiento. 
INFORMES

\section{Material y Métodos}

Se revisaron las historias clínicas de todos los pacientes ingresados en la Unidad de desintoxicación del Hospital Psiquiátrico Provincial de Madrid, durante los meses comprendidos entre marzo y septiembre de los años 1.994 y 1.999 con el fin de comparar las diferencias existentes entre los perfiles de desintoxicación. El número de ingresos durante dicho período de tiempo en 1994 fue de 142 y en 1.999 de 115 . Todos los ingresos eran voluntarios y llegaron derivados desde los centros de atención ambulatoria de drogodependientes de la Comunidad de Madrid, no participando los profesionales de la unidad en la indicación de este recurso.

Se elaboró un protocolo en el que se recogían variables relacionadas con características sociodemográficas, antecedentes médicos y familiares, historia de consumo, consumos durante el mes previo al ingreso para desintoxicación y tratamientos recibidos durante el mismo.

Se recogieron como datos sociodemográficos: edad, sexo, estado civil, hijos, convivencia, formación académica y situación laboral. Entre los antecedentes se identificó la presencia de infección por HIV y toma de antirretrovirales, VHC y VHB, los antecedentes familiares psiquiátricos y de consumo y los presencia de antecedentes penales. En la historia de consumo destacamos la droga, edad y vía de inicio de la adicción, aunque también identificamos otras sustancias de abuso y la edad y vía de inicio de consumo.

Dentro de las sustancias que fueron susceptibles de desintoxicación sólo se procesaron los datos de heroína, metadona, cocaína, benzodiacepinas y alcohol; así como el consumo medio en el último mes y la vía de administración. Dada la enorme frecuencia de consumo de dos o más sustancias se agruparon en diez categorías principales: (1)heroína, (2) heroína con cocaína y (3) heroína con otras combinaciones; (4) metadona, (5) metadona y sus combinaciones con cocaína, benzodiacepinas y alcohol; (6) cocaína y (7) cocaína con otras sustancias distintas de la heroína y la metadona; (8) benzodiacepinas y (9) su uso junto con el alcohol; y por último, (10) una categoría que agrupara al resto de sustancias.

Los datos recogidos de las historias clínicas se contrastaron con los informes de derivación de los centros ambulatorios de atención a los drogodependientes y con la entrevista familiar realizada al ingreso. Cuando existía discordancia se dio prioridad a la información recogida en los centros de atención ambulatoria, por ser éstos los que más contacto habían tenido con la historia de consumo del paciente.

En todos aquellos pacientes que no constaban serologías de VIH, VHB y VHB, se realizaron en el transcurso de la desintoxicación. 
Los datos de consumo al ingreso se contrastaron con detecciones urinarias de tóxicos y sólo se consideraron positivos aquellos en que existía coincidencia entre la información del paciente y la analítica.

El tratamiento recibido durante la desintoxicación se administró enmascarado en sellos de metilcelulosa y se ajustó a la sintomatología de abstinencia del paciente. Para desintoxicaciones de heroína se utilizaron agonistas y a -presinápticos; en las de metadona $\mathrm{a}_{2}$-presinápticos; en las de benzodiacepinas, diacepam o clonacepam; en las de alcohol, diacepam y vitaminoterapia (ácido fólico y complejo vitamínico $\mathrm{B}_{12} \mathrm{~B}_{6} \mathrm{~B}_{1}$ ) y en las de cocaína, benzodiacepinas si existía ansiedad e inhibidores selectivos de la recaptación de serotonina en caso de craving. Además se utilizaron otros fármacos como coadyuvantes en algunos síntomas de abstinencia sobre todo a opiáceos: analgésicos, propanolol, hipnóticos, benzodiacepinas y metoclopramida.

Para homogeneizar las dosis de benzodiacepinas se convirtieron los consumos de los distintos pacientes a su equivalencia en alprazolam, mientras que el tratamiento durante su ingreso se transformó en su equivalencia a diacepam, ya que la primera era la droga de abuso más usada entre los pacientes y el diacepam la benzodiacepina más prescrita. Para ello se utilizaron las equivalencias descritas por Kaplan y Sadock (10).

Su utilizaron las pruebas de la T de Student para comparar variables cuantitativas y la $\chi^{2}$ para las cualitativas. Los datos fueron analizados con el paquete estadístico SPSS.

\section{Resultados}

Las características sociodemográficas de las dos muestras aparecen en la tabla 1. Encontramos diferencias significativas en la edad media de los usuarios $(\mathrm{t}=5,55$; $\mathrm{p}<0,001)$, el estado civil $\left(\chi^{2}=9,33 ; \mathrm{p}>0,05\right)$ y en la situación laboral $\left(\chi^{2}=17,57 ; \mathrm{p}=0,001\right)$ donde destaca un mayor porcentaje de pacientes en situación laboral activa y de incapacitados en el año 1999.

Entre los antecedentes no encontramos diferencias significativas ni en la presencia de infección por VIH (43\% en 1994; 41,7\% en 1999); ni VHC (en 1994 fue del $57 \%$ y en 1999 del 73\%); ni VHB (52,8\% en 1994 por $60 \%$ en 1999). Sí existían diferencias en el número de enfermos que tomaban antiretrovirales (el 5,6 \% en 1994 y el $16,5 \%$ en $\left.1999 ; \chi^{2}=8,08 ; p<0,001\right)$. No existían diferencias significativas en los antecedentes familiares de consumo (el 42,3\% de los pacientes hospitalizados en 1994 tenían al menos un familiar de primer grado que había presentado algún tipo de adicción y en 1999 un 40,9\%), ni en la presencia de enfermedad mental familiar (11,3\% en 1994 y $13 \%$ en 1999). Tampoco encontramos diferencias significativas en la presencia de antecedentes penales, presentes en el 61,3\% de los desintoxicados en 1994 y en el 54,8\% de los de 1999. 
INFORMES

En la tabla 2 aparece un resumen de la historia de consumo, reflejando la droga inicial de adicción, su edad de inicio y el uso de la vía endovenosa al comienzo de la adicción. También aparecen dichos datos para las dos principales drogas de abuso en la nuestra población: heroína y cocaína.

Encontramos diferencias significativas en la droga de inicio $\left(\chi^{2}=14,58 ; \mathrm{p}<0,01\right)$, y en el consumo a lo largo de la historia de adicción de cocaína $\left(\chi^{2}=20,73 ; p<0,001\right)$, así como en la edad de inicio en el consumo de ésta última $(\mathrm{t}=2,51 ; \mathrm{p}<0,05)$. Otras drogas estudiadas fueron las benzodiacepinas y alcohol no encontrándose diferencias ni en la presencia de consumo ni en su edad de inicio.

En el año 1999 comenzamos a admitir en la unidad de desintoxicación a drogodependientes que utilizaban metadona en el contexto de tratamientos con agonistas opiáceos, dentro de un PMM, para desintoxicarse de otras sustancias, dicho tratamiento se inicia a una edad media de 30,87 años (d.e.=5,31; r[21-45]) y unas dosis medias de 99,38 mg (d.e.=60,41; r[4-300]).

Atendiendo a la desintoxicación propiamente dicha, el 100\% de los enfermos desintoxicados en 1994 fue para paso a un programa libre de drogas. Sin embargo en 1999, el 86,8\% fueron programas libres de droga y el 13,2\% planes de mantenimiento con metadona.

Más llamativo aún es el tipo de sustancias desintoxicadas (tabla 3). Todas las sustancias desintoxicadas durante el año 1994 correspondían a opiáceos, incluidas las agrupadas bajo el epígrafe otros (buprenorfina, codeína,..) y la desintoxicación de heroína aislada era prácticamente el 66,9\% de las desintoxicaciones. En 1999 la heroína sola deja de ser la sustancia más desintoxicada pasando al primer lugar la combinación de la misma con cocaína. Además aparece una mayor diversidad de sustancias desintoxicadas y aparecen pacientes que no consumen opiáceos (cocaína y benzodiacepinas). Mención aparte merecen las desintoxicaciones de metadona que suponen casi un 5\% y son producto de pacientes en PMM que pasan a PLD en 1999, la única desintoxicación de metadona realizada en 1994 fue un paciente cuya droga de abuso era ésta y no estaba en un programa de mantenimiento con agonistas.

Posteriormente, analizamos el consumo en el último mes de las cuatro sustancias que hemos considerado principales (heroína, cocaína, benzodiacepinas y alcohol), así como la dosis y vía de consumo.

En relación a la heroína encontramos diferencias significativas en cuanto a su uso (el 94,4\% de los desintoxicados la usaban durante el mes previo a la desintoxicación en 1994 y sólo el 58,3\% en 1999; $\chi^{2}=48,65 ; \mathrm{p}<0,001$ ), en cuanto a la vía de administración (en 1994 un 36,6\% usaban la vía intravenosa, por un 20,6\% en $1999 ; \chi^{2}=5,36 ; p<0,05$ ); pero no en la dosis utilizada (0,66 gr. de media en ambos períodos). 
Con respecto a la cocaína, existen diferencias en su frecuencia de uso (7\% en 1994 , frente al 63,5\% de $\left.1999 ; \chi^{2}=92,55 ; \mathrm{p}<0,001\right)$ y la dosis media de consumo en el mes previo (0,44 en 1994 y 0,98 en 1999; $\mathrm{t}=4,01 ; \mathrm{p}<0,001)$. No existen diferencias en el uso de la vía intravenosa (60\% en 1994 y 57,1\% en 1999).

La frecuencia de consumo tanto de benzodiacepinas ( $33,8 \%$ en 1994 y $53 \%$ en $\left.1999 ; \chi^{2}=9,63 ; p<0,01\right)$ como de alcohol $\left(10,6 \%\right.$ en 1994 y $21,9 \%$ en $1999 ; \chi^{2}=6,19$; $\mathrm{p}<0,05)$ es significativamente mayor en 1999. No encontramos sin embargo diferencias en la dosis utilizada (1994: benzodiacepinas $=6,52 \mathrm{mg}$ de alprazolam (d.e. $=6,21$ ), alcohol = 109,68 (d.e.=68,68). 1999: benzodiacepinas = 9,45 mg (d.e.=13,81), alcohol $=106,4 \mathrm{~g}($ d.e. $=49,06))$.

El $73 \%$ de los drogodependientes finalizaron el tratamiento de desintoxicación en 1999, con un tiempo de estancia en la unidad de 11,36 días (d.e. = 3,37). En 1994 finalizaron el 69,7\%, siendo el tiempo de desintoxicación 10,48 días (d.e. = 3,26).

Durante la desintoxicación, en 1994 recibieron tratamiento sustitutivo con metadona el 54,6\% de los pacientes, mientras en 1999 sólo el 34,5\% ( $\left.\chi^{2}=10,21 ; \mathrm{p}<0,001\right)$. En 1994 se trataron con a $a_{2}$-presinápticos a 43,4\% de los enfermos y el fármaco elegido fue la guanfacina ( $\mathrm{dmd}=2,57 \mathrm{mg}$, d.e.= 0,64); en 1999 se usó la clonidina en un 45,1\% $(\mathrm{dmd}=0,41 \mathrm{mg}$; d.e.= 0,15). Se usaron benzodiacepinas en ambos períodos en el 96,5\% de las desintoxicaciones pero a dosis significativamente mayores en 1999 (41,01 mg de diacepam, d.e.= 53,3, en 1999; por 28,71 mg, d.e. $=53,3$, en 1994; $\mathrm{t}=2,34 ; \mathrm{p}<0,05)$. Además en 1999 hubo un uso significativamente mayor analgésicos $\left(\chi^{2}=14,42 ; \mathrm{p}<0,001\right)$ y vitaminas $\left(\chi^{2}=22,44 ; \mathrm{p}<0,001\right)$ y menor de hipnóticos $\left(\chi^{2}=8,81 ; \mathrm{p}<0,01\right)$.

\section{Discusión}

El primer dato que merece nuestra atención es el envejecimiento de la población atendida que apoya la hipótesis de la cronicidad de las drogodependencias, con recaídas más o menos frecuentes que llevan a nuevas desintoxicaciones cada vez a mayor edad. Este envejecimiento justifica el cambio en el estado civil. Por otra parte, el llamativo aumento de pacientes laboralmente activos apunta a lo ya señalado en la literatura: los adictos a drogas no opiáceos, más numerosos en el año 1999, se mantienen más integrados socialmente que los heroinómanos(11) y los pacientes en PMM son capaces de compaginar este tratamiento con una actividad laboral $(12,13)$.

Un dato relativamente alentador es el mantenimiento de la prevalencia de la serología VIH (+), que confirma el papel de los antiretrovirales en la mejora pronóstica de estos enfermos y el beneficio que los programas de prevención de daño, incluido el PMM, pueden jugar en frenar la incidencia de esta infección entre consumidores de drogas vía parenteral(14). Quizás, una vez conseguido este importante reto, haya que potenciar otros programas de salud en pacientes adictos dirigidos a enfermedades de gran prevalencia 
INFORMES

en este grupo, como son: la hepatitis $\mathrm{C}$, en donde sí encontramos un importante aumento porcentual en nuestros resultados y, la tuberculosis que está en vías de convertirse en un grave problema de salud publica, si bien en nuestro estudio no recogimos datos que corroboren este supuesto aumento en su incidencia.

Los resultados demuestran un cambio significativo en la droga de inicio de la adicción y concuerdan con los datos de la Agencia Antidroga de la Comunidad de Madrid(4) y los hallazgos de la literatura $(15,16)$. La heroína pierde peso como droga única, mientras que lo gana la cocaína en solitario o combinada con heroína. La cocaína como droga de abuso adquiere un papel significativo respecto al año 1994, con un claro incremento por vía i.v. Menos congruente resulta la mayor edad de inicio del consumo de drogas reportada en 1999 que, aunque con una $\mathrm{p}<0,05$, contrasta con los hallazgos de las últimas publicaciones donde se informa de una reducción de la edad de inicio del consumo(17). Como ya han señalado otros autores(18), la recogida de datos a partir de la información que facilita el paciente siempre introduce un sesgo, y probablemente más, en el tema del consumo de sustancias.

El cambio en el tipo de sustancias a desintoxicar experimentado por la población de la Unidad de Desintoxicación refleja el cambio de tendencia de consumo en la calle. El aumento de pacientes consumidores de metadona obedece a la admisión en la UDH a partir de 1999 de pacientes en PMM para desintoxicación de otras sustancias y/o de metadona, sin embargo este aumento del $5 \%$ es proporcionalmente muy inferior al experimentado por el número de plazas de metadona en la Comunidad de Madrid, que como ya se ha señalado se multiplicó en el mismo periodo por más de 30 . Esa desproporción puede indicar que sólo una pequeña parte de los pacientes en PMM presentan adicción a otras sustancias, o bien que la mayor parte de ellos pueden abandonar la terapia sustitutiva sin pasar por una unidad hospitalaria o, la más pesimista de las posibilidades, que las plazas existentes para ese fin están muy por debajo de las necesidades. La media de edad más alta de estos pacientes obedece a que son pacientes que han fracasado en intentos previos de mantenerse en PLD y llegan al PMM después de años de dependencia de heroína.

La heroína sola deja de ser la principal droga a desintoxicar y se reduce significativamente su consumo respecto a 1994, diversificándose las drogas consumidas y desintoxicadas. El multiconsumo y la multidependencia se convierten en norma y la cocaína aparece en el $60 \%$ de los casos, con un aumento significativo de la dosis media consumida, que pasa de $0.5 \mathrm{~g}$ /día a una dosis próxima a $1 \mathrm{~g}$ /día. También es llamativo y alarmante el aumento de la adicción a benzodiacepinas, fundamentalmente alprazolam, y alcohol en consumidores de otras drogas, principalmente metadona y cocaína. Un $20 \%$ más de los pacientes presentaron dependencia de benzodiacepinas y el número de dependientes del alcohol prácticamente doblaba el de 1994. 
El dato positivo en cuanto a la evolución del consumo es la tendencia estadísticamente significativa al uso de la vía inhalada frente a la predominancia de la vía intravenosa en 1994. Este hallazgo consistente con el de otros lugares(19-21), refleja nuevamente el éxito de los programas de reducción de daño, pero también los avatares del mercado de drogas ilegales.

Los resultados sobre éxito y estancia media de las desintoxicaciones realizadas en 1994 y 1999, no arrojan diferencias significativas. El aumento de las desintoxicaciones de alcohol, benzodiacepinas y metadona, que habitualmente requieren periodos más largos que la desintoxicación de heroína sola, nos indujeron a sospechar un alargamiento de la estancia media que los resultados no confirman. Sin embargo, un análisis más detallado demuestra el incremento del numero de desintoxicaciones de cocaína sola, con estancia habitual de siete días, parecen haber contrarrestado el aumento.

Finalmente, en cuanto al tratamiento farmacológico lo más destacable resulta la reducción de la frecuencia de uso de metadona como tratamiento sustitutivo en las desintoxicaciones, el mantenimiento de los $\mathrm{a}_{2}$-presinápticos, aunque con el cambio de guanfacina a clonidina provocado por la retirada del mercado de la primera, el mantenimiento de la frecuencia de indicación de benzodiacepinas aunque con un importante incremento de la dosis media utilizada durante 1999. También aumentó la prescripción de analgésicos y de vitaminas. En nuestra opinión, todos estos cambios pueden atribuirse a la reducción del número de desintoxicaciones puras de heroína y al aumento de desintoxicaciones de benzodiacepinas, cocaína y alcohol.

\section{Conclusiones}

El perfil de pacientes que ingresaron en la Unidad de Desintoxicación del Hospital Psiquiátrico de Madrid durante 1999 varió significativamente respecto al año 1994, tanto en las características sociodemográficas como en el perfil toxicológico. La población era de más edad y presentaba una clara diversificación del consumo de tóxicos, con una predominancia de los multidrogodependientes frente a los monodependientes y de la vía inhalada de consumo frente a la intravenosa. La desintoxicación de alcohol, benzodiacepinas, heroína y metadona no supuso un cambio en el tipo de fármacos empleados en el tratamiento, salvo el uso de vitaminoterapia, pero sí un incremento de las dosis de benzodiacepinas utilizadas. Se requerirían nuevos estudios para definir la estancia media del nuevo perfil, que en el presente trabajo se ve engañosamente conservada por los consumidores de cocaína, y las necesidades reales de plazas de desintoxicación para pacientes en mantenimiento con agonistas opiáceos. 
INFORMES

Tabla 1. Características sociodemográficas.

\begin{tabular}{|c|c|c|}
\hline & $\begin{array}{c}1994 \\
\left(\mathrm{n}_{1}=142\right)\end{array}$ & $\begin{array}{c}1999 \\
\left(n_{2}=115\right)\end{array}$ \\
\hline$\overline{\operatorname{Edad}(* * *)}$ & 29,1 & 33,22 \\
\hline Rango & $16-48$ & $17-50$ \\
\hline D.E. & 6,09 & 5,68 \\
\hline Sexo & & \\
\hline Hombre & $78,2 \%$ & $79,1 \%$ \\
\hline Mujer & $21,8 \%$ & $20,9 \%$ \\
\hline Estado civil $(*)$ & & \\
\hline Soltero & $70,4 \%$ & $53,9 \%$ \\
\hline Casado / en pareja & $16,2 \%$ & $19,1 \%$ \\
\hline Separado / divorciado & $12 \%$ & $25,2 \%$ \\
\hline Viudo & $1,4 \%$ & $1,7 \%$ \\
\hline Hijos & & \\
\hline Sí & $35,9 \%$ & $43,5 \%$ \\
\hline No & $64,1 \%$ & $54,8 \%$ \\
\hline Conviven los hijos & & \\
\hline Sí & $47,07 \%$ & $34,02 \%$ \\
\hline No & $52,92 \%$ & $65,97 \%$ \\
\hline Convivencia & & \\
\hline Solo & $4,2 \%$ & $10,4 \%$ \\
\hline Familia propia & $15,5 \%$ & $20,9 \%$ \\
\hline Familia de origen & $73,2 \%$ & $60 \%$ \\
\hline Otros & $7 \%$ & $7,8 \%$ \\
\hline Formación académica & & \\
\hline Sin estudios & $38 \%$ & $23,5 \%$ \\
\hline Graduado escolar & $47,9 \%$ & $53,9 \%$ \\
\hline$B U P$ / COU / FP & $13,4 \%$ & $16,5 \%$ \\
\hline Universitarios & $0,7 \%$ & $3,5 \%$ \\
\hline Situación laboral $(* * *)$ & & \\
\hline En activo & $13,4 \%$ & $20,9 \%$ \\
\hline Parado con subsidio & $9,9 \%$ & $9,6 \%$ \\
\hline Parado sin subsidio & $74,6 \%$ & $55,7 \%$ \\
\hline Incapacitado & $2,1 \%$ & $13,9 \%$ \\
\hline
\end{tabular}

$(* * *) \mathrm{p} £ 0,001$

(*) $\mathrm{p}<0,05$ 
Tabla 2 .Historia de consumo

\begin{tabular}{|c|c|c|}
\hline & 1994 & 1999 \\
\hline \multicolumn{3}{|c|}{ Droga inicial de adicción } \\
\hline $\begin{array}{l}\text { Tipo de sustancia }(* *) \\
\text { Heroína } \\
\text { Cocaína } \\
\text { Ambas } \\
\text { Otras }\end{array}$ & $\begin{array}{c}97,2 \% \\
- \\
2,1 \% \\
0,7 \% \\
\end{array}$ & $\begin{array}{c}84,3 \% \\
5,2 \% \\
8,7 \% \\
1,7 \% \\
\end{array}$ \\
\hline $\begin{array}{l}\text { Vía I.V. } \\
\text { Sí } \\
\text { No }\end{array}$ & $\begin{array}{r}49,3 \% \\
50,7 \% \\
\end{array}$ & $\begin{array}{r}44,3 \% \\
55,7 \% \\
\end{array}$ \\
\hline Edad de inicio & $\begin{array}{c}18,83 \\
\text { s.d. }=5,06\end{array}$ & $\begin{array}{c}19,76 \\
\text { s.d. }=5,33\end{array}$ \\
\hline \multicolumn{3}{|c|}{ Heroína } \\
\hline $\begin{array}{l}\text { Consumo } \\
\text { Sí } \\
\text { No }\end{array}$ & $\begin{array}{r}99,3 \% \\
0,7 \%\end{array}$ & $\begin{array}{c}94,8 \% \\
5,2 \%\end{array}$ \\
\hline $\begin{array}{l}\text { Vía I.V. } \\
\text { Sí } \\
\text { No }\end{array}$ & $\begin{array}{r}49,3 \% \\
50,7 \%\end{array}$ & $\begin{array}{l}40 \% \\
60 \%\end{array}$ \\
\hline Edad de inicio & $\begin{array}{c}18,84 \\
\text { s.d. }=5,06\end{array}$ & $\begin{array}{c}19,50 \\
\text { s.d. }=5,42\end{array}$ \\
\hline \multicolumn{3}{|c|}{ Cocaína } \\
\hline $\begin{array}{l}\text { Consumo }(* * *) \\
\text { Sí } \\
\text { No }\end{array}$ & $\begin{array}{l}70,2 \% \\
29,8 \%\end{array}$ & $\begin{array}{c}93 \% \\
7 \%\end{array}$ \\
\hline $\begin{array}{l}\text { Vía I.V. } \\
\text { Sí } \\
\text { No } \\
\end{array}$ & $\begin{array}{l}28,2 \% \\
71,8 \% \\
\end{array}$ & $\begin{array}{r}35,7 \% \\
64,3 \% \\
\end{array}$ \\
\hline Edad de inicio $(*)$ & $\begin{array}{c}18,88 \\
\text { s.d. }=4,41\end{array}$ & $\begin{array}{c}20,65 \\
\text { s.d. }=5,37\end{array}$ \\
\hline
\end{tabular}
(*) $\mathrm{p}<0,05$
(**) $\mathrm{p}<0,01$
$(* * *) \mathrm{p}<0,001$ 
INFORMES

Tabla 3. Sustancias desintoxicadas.

\begin{tabular}{|l|c|c|c|c|}
\hline \multirow{2}{*}{} & $\mathbf{1 9 9 4}$ & \multicolumn{3}{|c|}{$\mathbf{1 9 9 9}$} \\
\cline { 2 - 5 } & TOTAL & PLD & PMM & TOTAL \\
\hline Heroína & $66,9 \%$ & $52 \%$ & - & $18,3 \%$ \\
Heroína y cocaína & $6,3 \%$ & $17 \%$ & - & $25,2 \%$ \\
Heroína y otras & $21,1 \%$ & $18,8 \%$ & - & $10,4 \%$ \\
Metadona & - & $1,3 \%$ & - & $2,6 \%$ \\
Cocaína yotras & $0,7 \%$ & $3,1 \%$ & - & $6,1 \%$ \\
Cocaína y otras & - & $1,8 \%$ & $17,6 \%$ & $8,7 \%$ \\
Benzodiacepinas & - & $1,3 \%$ & $47,1 \%$ & $16,5 \%$ \\
Benzodiacepinas y otras & - & $0,4 \%$ & $20,6 \%$ & $7 \%$ \\
Otras & - & - & $11,8 \%$ & $3,5 \%$ \\
\hline
\end{tabular}

\section{BIBLIOGRAFÍA:}

1. Rosenbaum, M. et al, "Treatment as harm reduction, defunding as harm maximization", $J$ Psychoactive Drugs, 1996, 28,pp. 241-9.

2. Márkez Alonso, I.; Póo Ruiz, M., "Nuevos modelos de intervención: disminución de riesgos y programas de mantenimiento", Psiquiatría Pública, 1998,pp. 378-388.

3. Plan Regional sobre Drogas, Memoria 1994, Madrid, Comunidad de Madrid,1995.

4. Agencia Antidroga de la Comunidad de Madrid, Memoria 1999, Madrid, Comunidad de Madrid, 2000.

5. Gollnish, G., "Múltiple predictors of illicit drug use in methadone maintenance clients", Addict Behav 1997, 22,pp. 353-66.

6. Markez, I.; Pinilla, E.; Aizpuru, A., "Control de drogas en orina: incidencia y utilidad en PMM", Adicciones, 1998, 10, pp. 131-7.

7. Joseph, H.; Appel, P., "Alcoholism and methadone treatment consequences for the patient and program", Am J Drug Alcohol Abuse, 1985, 11, pp. 37-53.

8. Grella, C.E.; Anglin, M.D.; Wugalter, S.E., "Patterns and predictors of cocaine and crack use by clients in standard and enhanced methadone maintenances treatment", Am J Drug Alcohol Abuse, 1997, 23, pp. 15-42.

9. Sopelana, P.; Bautista, L.; Diéguez, A., "Consumo de cocaína en los drogodependientes de heroína", Archivos de Neurobiología, 1993, 2, pp. 82-8.

10. Kaplan, H.I.; Sadock, B.J., Pocket Handbook of Psychiatric Drug Treatment, Baltimore, Williams \& Wilkins, 1993.

11. Jaffe, J.H., "Cocaine-related disorders". En: Sadock, B.J.; Sadock, V.A. ed. Kaplan y Sadock's Comprehensive Textbook of Psychiatry, Filadelfia, Lippincott Williams and Wilkins, 1999, pp. 999-1015.

12. Bertschy, G., "Methadone maintenance treatment: an update", Eur Arch Psychiatry Clin Neurosci, 1995, 245, pp. 114-24.

13. Farell, M. et al., "Methadone maintenance treatment in opiate dependence: a review", $B M J$, 1994, 309, pp. 997-1001. 
14. Marsch, L.A., "The efficacy of methadone maintenance interventions in reducing illicit opiate use, HIV risk behaviour and criminality: a metaanalysis", Addiction, 1998, 93, pp. 515-32.

15. Bauman, A.; Phongsavan, P., "Epidemiology of substance use in adolescence: prevalence, trends and policy implications", Drug Alcohol Depend, 1999, 55, pp.187-207.

16. Bravender, T.; Knight, J.R. "Recent patterns of use and associated risks of illicit drug use in adolescents", Curr Opin Pediatr ,1998, 10, pp. 344-9.

17. Gilvarry, E., "Substance abuse in young people", J Child Psychol Psychiatry, 2000, 41, pp.55-80.

18. Harrison, E.R.; Haaga, J.; Richards, T., "Self-reported drug use data: what do they reveal?", Am J Drug Alcohol Abuse, 1993, 19, pp. 423-41.

19. Iraurgi Castillo, I.; Vielva Pérez, I., "Cambio en la pauta de administración de drogas en la comunidad autónoma vasca. Evolución durante el periodo 1991-1996”. Rev Esp Salud Pública, 2000, 74, pp.119-29.

20. Hagan, H. et al., "Reduced injection frequency and increased entry and retention in drug treatment associated with needle-exchange participation in Seattle drug injectors", $J$ Subst Abuse Treat, 2000, 19, pp. 245-52.

21. de la Fuente, L. Et al., "The transition from injecting to smoking heroin in three Spanish cities. The Spanish Group for the Study of the Route of Heroin Administration", Addiction, 1997,92, pp.1749-63.

*Psiquiatras

Hospital Psiquiátrico de Madrid

Correspondencia: Ana González Rodríguez

Hospital Psiquiátrico de Madrid

Carretera de Colmenar, km. 13.800

28049 Madrid

Fecha de recepción: 20-02-02 